\title{
TeraHertz inspections of painted steel samples
}

\author{
Ilaria Catapano \\ Institute for Electromagnetic Sensing of \\ the Environment - National Research \\ Council of Italy, via Diocleziano 328, \\ 80124 Napoli, Italy \\ catapano.i@irea.cnr.it
}

Thibaud Toullier

Univ Gustave Eiffel, Inria, COSYS-SII, I4S Team, F-44344 Bouguenais, France

thibaud.toullier@univ-eiffel.fr

\author{
Giovanni Ludeno \\ Institute for Electromagnetic Sensing of \\ the Environment - National Research \\ Council of Italy, via Diocleziano 328, \\ 80124 Napoli, Italy \\ ludeno.g@irea.cnr.it
}

Jean Dumoulin

Univ Gustave Eiffel, Inria, COSYS-SII,

I4S Team, F-44344 Bouguenais,

France

jean.dumoulin@univ-eiffel.fr

\author{
Francesco Soldovieri \\ Institute for Electromagnetic Sensing of \\ the Environment - National Research \\ Council of Italy, via Diocleziano 328, \\ 80124 Napoli, Italy \\ soldovieri.f@irea.cnr.it
}

\begin{abstract}
Aerospace industry needs accurate coating thickness measurement as well as adhesion testing for preventing the corrosion of wear of metal substrates. Recently, a constant attention is focused towards the potentialities offered by non-invasive sensing techniques and their technological advancements. This communication deals with time of flight THz imaging, enhanced by a noise filtering procedure based on the Singular Value Decomposition of the data matrix. In this work, THz imaging is exploited to analyse painted steel samples in order to detect paint coating layers and provide images of them and of the interface between the coating layer and the steel substrate.
\end{abstract}

Keywords-THz imaging, Non-destructive inspection, coating analysis.

\section{INTRODUCTION}

Paint coatings are applied to aerospace materials for appearance and protection against environmental factors. Therefore, adhesion tests as well as analysis devoted to establish the integrity of the coating are part of quality and security controls. In this framework, recently there is a research effort aiming at making advancements in new nondestructive inspection technologies integrated with the technologies usually exploited in the quality control during the production and the operability of the aeronautic materials.

One of the sensing technologies attracting now interest regards the use of Terahertz systems, i.e. electromagnetic imaging instrumentations working in the frequency range from $0.1 \mathrm{THz}$ to $10 \mathrm{THz}$ (free-space wavelength ranging from $30 \mu \mathrm{m}$ to $3 \mathrm{~mm}$ ) [1]. THz waves are, indeed, non-ionizing radiations able to penetrate non-metallic and optical opaque media; hence, allow non-destructive, contact-free and safe surveys providing images, with a sub-millimetre spatial resolution, of the inner structure of the investigated object. Examples assessing $\mathrm{THz}$ potentialities for aerospace applications are given in [2-5].

This communication presents the results of $\mathrm{THz}$ surveys recently carried out by means of the system available at the Institute for Electromagnetic Sensing of the Environment (IREA) - National Research Council of Italy (CNR) and regarding samples prepared at the Metallic Structures and Cables Laboratory of University Gustave Eiffel. The samples are coated steel plates in a good status. The analysis of the $\mathrm{THz}$ data is still on going, and the results presented in this extended abstract are preliminary and will be updated in the final paper.

\section{MATERIAL AND METHODS}

A. THz system

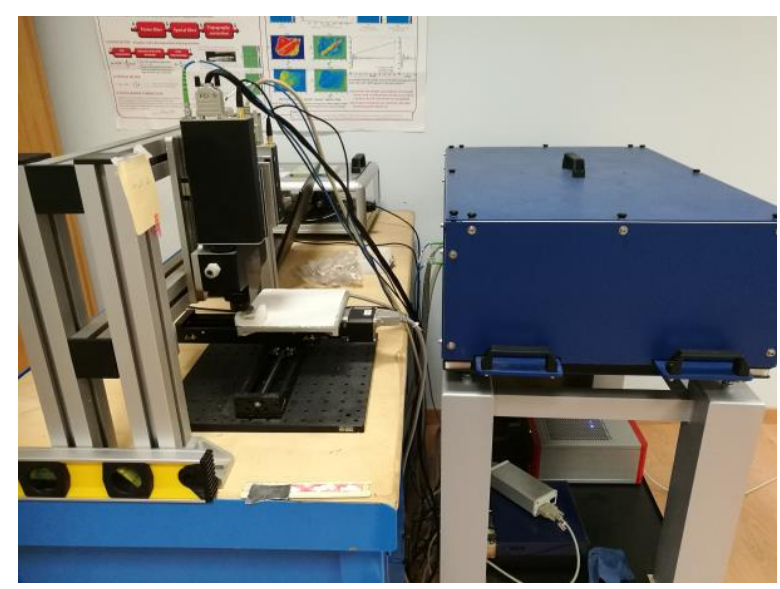

Figure 1. Zomega FICO THz system

$\mathrm{THz}$ surveys have been carried out by means of the Zomega FICO system, shown in Fig. 1. The system is a time domain spectrometer generating and detecting $\mathrm{THz}$ pulses, with full waveform sampling rates up to $500 \mathrm{~Hz}$.

The pump source is an external $1.5 \mu \mathrm{m}$ pulsed laser, which is coupled with the system optically and shielded by an aluminum case. The emitter consists of a photoconductive dipole antenna fabricated on a LT-GaAs wafer and a $25-\mathrm{mm}$ FL TPX lens is used to collimate the $\mathrm{THz}$ beam out of the module. The receiver uses also a TPX lens to drive the incoming $\mathrm{THz}$ radiation on an EO detection crystal (GaAs) collinearly with the optical probe beam. Emitter and receiver are connected to the FiCO system by means of optic fibers, so they are reconfigurable in transmission and normal reflection mode.

The delay between pump and probe is controlled by the rotary delay line (RODL) internal to the emitter module and by the timing stage. The RODL allows for constant scanning of $100 \mathrm{ps}$, which is the observation time window. The timing stage allows the shift of the time window along a time scan range of $1 \mathrm{~ns}$, according to the length of the transmittersample-receiver propagation path.

Imaging surveys have been performed by using the normal reflection module, which holds emitter and receiver and is mounted on an automatic positioning system (see Fig. 1) enabling a planar scan. The maximum allowable scan area is $150 \times 150 \mathrm{~mm}^{2}$, with a minimum spatial step equal to $120 \mu \mathrm{m}$. The vertical position of the sample is arranged manually in order to achieve the focal distance between the sample and the 
normal reflection module. The normal reflection configuration is enabled thanks to a 50-mm TPX lens, so that measurements are possible with a $\mathrm{THz}$ focused beam having a $0^{\circ}$ incidence angle.

FiCO system has been used in normal environmental conditions (without purging with $\mathrm{N}_{2}$ ) and in high-speed mode. The use of high-speed mode entails that, for each measurement point, the stored $\mathrm{THz}$ signal is obtained by averaging a reduced number of waveforms. As a consequence, the gathered data are affected by the environmental conditions and the effective frequency range ranges from $40 \mathrm{GHz}$ up to $1.16 \mathrm{THz}$. This means, that in the analysis below presented, the spectrum portion involving frequencies higher than 1.7 $\mathrm{THz}$ has not been considered.

\section{B. Data processing procedure}

The first step of the data processing is a filtering procedure devoted to reduce the detrimental effect of the environmental noise. Such a procedure is detailed in [5] and it is based on the computation of the Singular Value Decomposition (SVD) [6] of the data matrix. The SVD-based de-noising procedure is capable of managing data filtering without affecting the spatial resolution capabilities.

The subsequent steps are those commonly adopted in time of flight imaging. Specifically, they exploit the relationship existing among the spatial distance $d$ from $\mathrm{THz}$ probes to the detected electromagnetic discontinuity, and the time of flight $t$, i.e. the time that the waveform employs to propagate along the path emitter-discontinuity-receiver, through the wave propagation velocity $v$ inside the investigated materials:

$$
t=\frac{2 * d}{v}
$$

Accordingly, each collected signal is plotted as a function of the time (A-scan) and data referred to the same measurement line are represented as a space-time image, referred as a $\mathrm{THz}$ radargram. This latter provides a crosssection representation giving information on the location and thickness of the inner layers. Data collected on the entire scan area are exploited to obtain a three-dimensional (3D) characterization of the object, which is commonly visualized by means of $2 \mathrm{D}$ images showing the investigated volume at different constant depths.

Being the constant depth slice visualization affected by the topography of the material layers and by localized electromagnetic anomalies, it allows a reliable view only when we have perfectly flat interfaces between homogeneous materials. To overcome this drawback while avoiding complex peak alignment procedure, herein the imaging of surface and the sub-surface material interfaces is performed by: 1) selecting the portions of the time window where the reflection occurs; 2) generating false color images obtained as the pixel by pixel representation of the maximum signal amplitude into the considered time window portion.

\section{Investigated Samples}

The analysed samples are depicted in Figure 2. Both are made of $95 \mathrm{~mm}$ x $95 \mathrm{~mm}$ square steel plates coated with protective offshore paint to prevent steel structure from corrosion. The steel plates were not submitted to corrosion accelerating testing and were covered by using different

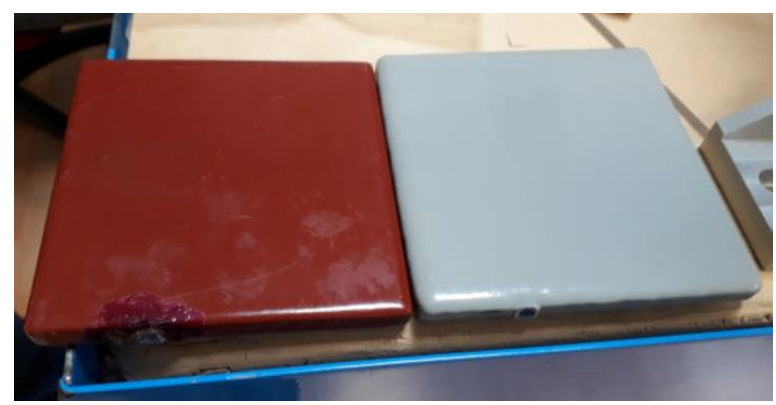

Figure 2. View of the inspected Red and Gray samples

coating paints. For both the samples, THz data were gathered by putting the normal reflection module $6.0 \mathrm{~mm}$ far from the sample face shown in Fig. 2 and by scanning a $100 \mathrm{~mm} \times 100$ $\mathrm{m}$ area with a $0.5 \mathrm{~mm}$ spatial offset along $\mathrm{x}$ - and $\mathrm{y}$-axis. In the following, the samples are named red sample and grey sample according to the colour of the external coating.

\section{RESULTS}

\section{A Red sample}

Figure 3 shows three representative radargrams and for each one the A-scans corresponding to the dashed white lines. The radargrams permit to detect three interfaces:

- the first one arises on average at 43 ps and denotes the painted surface of the sample;

- the second interface occurs at 46 ps and indicates the presence of a subsurface paint layer;

- the third interface is on average at 55 ps and corresponds to the steel plate.

The presence of three main peaks is corroborated by the A-scans, which also make evident that the first two peaks have comparable amplitude, while the amplitude of the third one denotes that the steel reflectivity is attenuated due to the overlapping paint layers.

Figure 3 also suggests that the interfaces referred to the paint layers are flat except for the sample boundary where, as
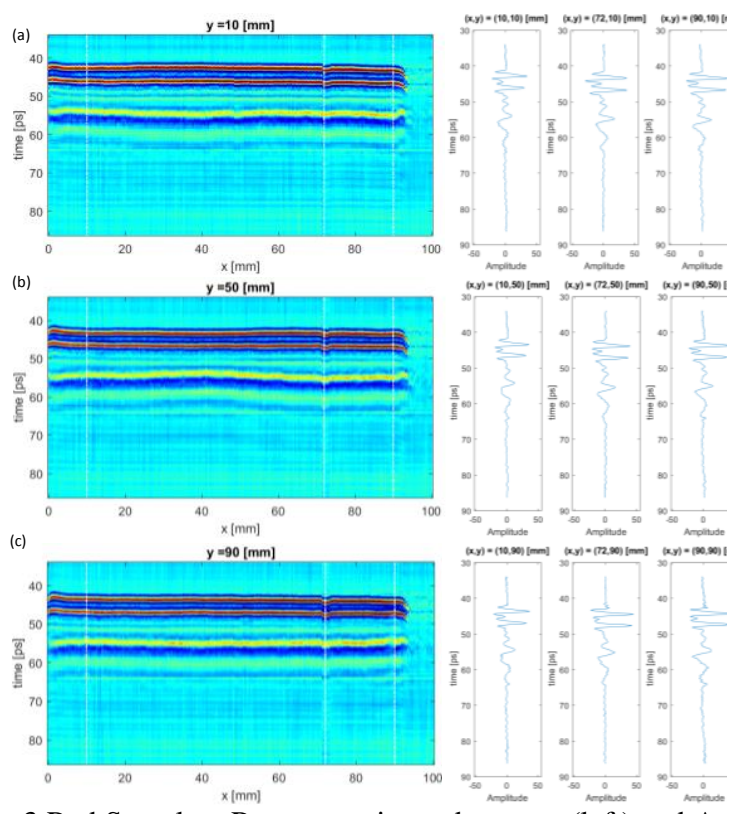

Figure 3 Red Sample - Representative radargrams (left) and Ascans (right) referred to: (a) $\mathrm{y}=10 \mathrm{~mm}$; (b) $\mathrm{y}=50 \mathrm{~mm}$; (c) $\mathrm{y}=$ $90 \mathrm{~mm}$. 

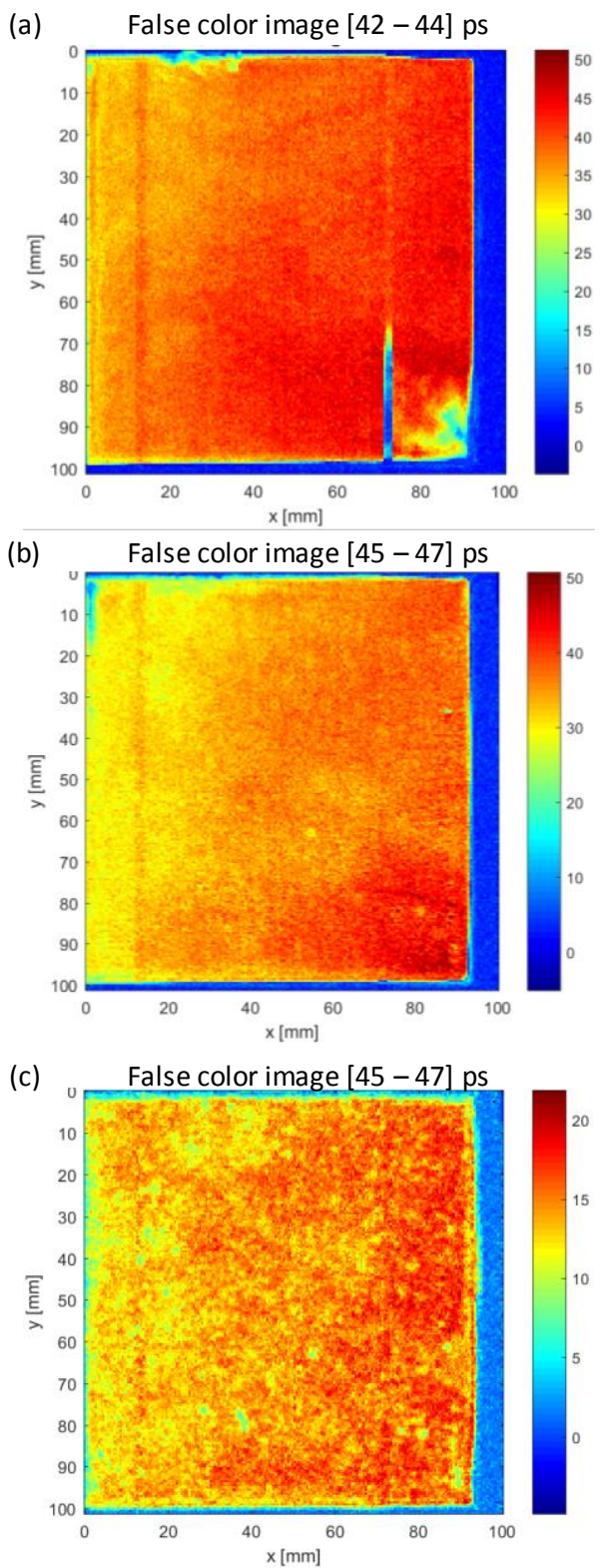

Figure 4 Red Sample - False color images of the material interfaces

expected, they are curved. Conversely, the plate surface appears slightly curl. Moreover, in all the radargrams, collected along measurement lines parallel to the $\mathrm{x}$-axis, a slight depression occurs at $\mathrm{x}=72 \mathrm{~mm}$. This anomaly implies a delay of the reflected signal about equal to $0.4 \mathrm{ps}$.

$\mathrm{THz}$ images of the detected interfaces are given in Figure 4 , which shows the false colour images corresponding to the time window portions [42-44] ps; [45-47] ps; [54-56] ps and computed as described in Section II-A.

Based on these images one may infer that: i) the depression at $\mathrm{x}=72 \mathrm{~mm}$ is ascribable to an irregularity of the surface paint layer (see upper panel); ii) the paint layers have a flat topography but their reflectivity is not uniform on the entire sample area; iii) the steel plate surface appears rough and exhibits localized non-homogeneities.

\section{B Gray sample}

Figure 5 shows three representative radargrams and for each one the A-scans corresponding to the dashed white lines.
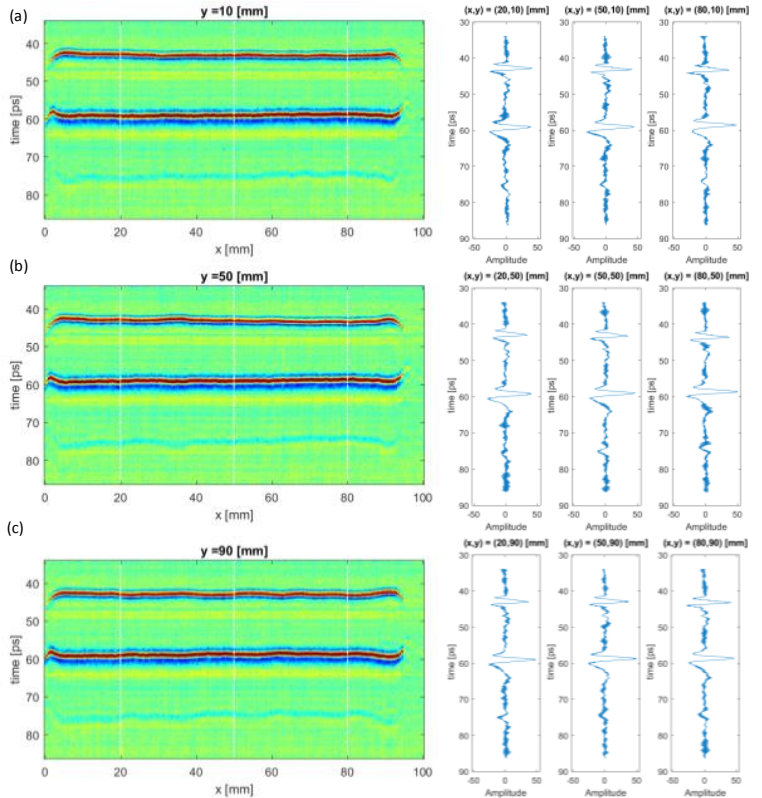

Figure 5 Gray Sample - Representative radargrams (left) and Ascans (right) referred to: (a) $\mathrm{y}=10 \mathrm{~mm}$; (b) $\mathrm{y}=50 \mathrm{~mm}$; (c) $\mathrm{y}=$ $90 \mathrm{~mm}$.

For this sample, the radargrams show the presence of two clearly visible interfaces occurring on average at 43 ps and 59 ps, which could be ascribable to the paint layers, and a third less evident one on average at 75 ps maybe corresponding to the paint-steel interface.

It is worth nothing that the first interface occurs at the same time instant for both grey and red samples, while the second interface occurs later in the case of grey sample. This suggest that the dielectric permittivity of the grey paint is lower than that of the red one. Such a hypothesis is supported by the Ascans, which make also clear that the third peak has a negative maximum amplitude.

According to this observation, Figure 6 depicts the false colour image referred to the time window portions [40-50] ps; [51-62] ps, [70-80] ps, where the image referred to the third sub-window is the pixel by pixel representation of the minimum signal amplitude instead of the maximum, as for the other two ones.

The images in Fig. 6 denote that: i) the paint layers have substantially flat topography; ii) the surface paint layer has a quite uniform reflectivity on the entire area of the sample, while the reflectivity of the inner paint layer appears on average higher for $\mathrm{x}$ ranging from $40 \mathrm{~mm}$ to $80 \mathrm{~mm}$, whatever the $y$ value; iii) the reflectivity of the steel is strongly attenuated by the overlapping paint layers and, as for the red sample, it appears curls.

\section{CONCLUSIONS}

$\mathrm{THz}$ imaging is worth being considered among the other non-invasive testing technologies in the aerospace industry especially as concerns the coating analysis.

This communication has reported some preliminary results referred to on-going $\mathrm{THz}$ analysis of painted steel plates. Since the study is not completed yet, result updates are foreseeable and, in that case, they will provide in the final paper. 

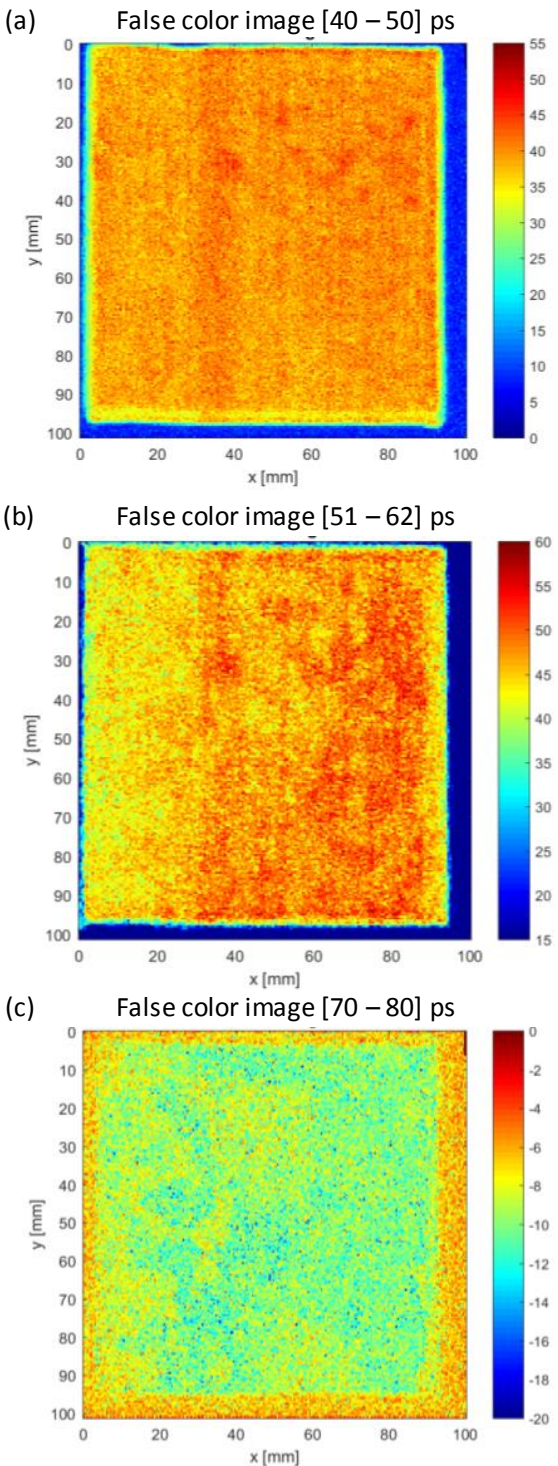

Figure 6 Gray Sample - False color images of the material interfaces

\section{ACKNOWLEDGMENT}

Authors wish to thank Dr. Laurent Gaillet from Metallic Structures and Cables Laboratory at Univ. Gustave Eiffel for providing samples for this study.

\section{REFERENCES}

[1] S. Wang and X. C. Zhang, Pulsed terahertz tomography, J. of Physics D: Applied Physics, vol 37, pp. R1-R36, Jan. 2004

[2] Zimdars, David, et al. "Technology and applications of terahertz imaging non - destructive examination: inspection of space shuttle sprayed on foam insulation." AIP conference proceedings. Vol. 760. No. 1. American Institute of Physics, 2005.

[3] Quast, Holger, Andreas Keil, and Torsten Löffler. "Investigation of foam and glass fiber structures used in aerospace applications by allelectronic 3D Terahertz imaging." 35th international conference on infrared, millimeter, and terahertz waves. IEEE, 2010.

[4] Bohn, Matthew J., and Douglas T. Petkie. "Terahertz applications in the aerospace industry." Handbook of Terahertz Technology for Imaging, Sensing and Communications. Woodhead Publishing, 2013. 510-546.

[5] Catapano, I., Soldovieri, F., Mazzola, L., \& Toscano, C. (2017). THz Imaging as a method to detect Defects of aeronautical coatings. Journal of Infrared, Millimeter, and Terahertz Waves, 38(10), 1264-1277.

[6] M. Bertero and P. Boccacci (1998), Introduction to Inverse Problems in Imaging, Bristol: Institute of Physics Publishing.. 